\title{
Statistical estimation of nonstationary Gaussian processes with long-range dependence and intermittency
}

\author{
Jiti $\mathrm{Gao}^{\mathrm{a}, *}$, Vo $\mathrm{Anh}^{\mathrm{b}}$, Chris Heyde $\mathrm{c}^{\mathrm{c}, \mathrm{d}}$ \\ ${ }^{a}$ The University of Western Australia, Perth, Australia \\ ${ }^{\mathrm{b}}$ Queensland University of Technology, Brisbane, Australia \\ ${ }^{\mathrm{c} C o l u m b i a}$ University, New York, USA \\ ${ }^{\mathrm{d}}$ The Australian National University, Canberra, Australia
}

Received 13 December 1999; received in revised form 23 October 2001; accepted 31 January 2002

\begin{abstract}
This paper considers statistical inference for nonstationary Gaussian processes with long-range dependence and intermittency. The existence of such a process has been established by Anh et al. (J. Statist. Plann. Inference 80 (1999) 95-110). We systematically consider the case where the spectral density of nonstationary Gaussian processes with stationary increments is of a general and flexible form. The spectral density function of fRBm is thus a special case of this general form. A continuous version of the Gauss-Whittle objective function is proposed. Estimation procedures for the parameters involved in the spectral density function are then investigated. Both the consistency and the asymptotic normality of the estimators of the parameters are established. In addition, a real example is presented to demonstrate the applicability of the estimation procedures. (c) 2002 Published by Elsevier Science B.V.
\end{abstract}

Keywords: Asymptotic theory; Fractional Riesz-Bessel motion; Nonstationary process; Long-range dependence; Statistical estimation

\section{Introduction}

Many recent studies have indicated that data in a large number of fields display long-range dependence (LRD) (Beran, 1994; Baillie and King, 1996; Anh and Heyde, 1999). A basic process which has commonly been used to model LRD is fractional

\footnotetext{
* Corresponding author. School of Mathematics and Statistics, The University of Western Australia, 6009 Crawley, WA, Australia. Tel: +618-9380-3354; fax: +618-9380-1028.

E-mail address: jiti@maths.uwa.edu.au (J. Gao).
} 
Brownian motion $(\mathrm{fBm}) B_{H}$ with Hurst index $\frac{1}{2}<H<1$. This is a Gaussian process which has stationary increments and spectral density of the form

$$
\phi_{1}(\omega)=\frac{c}{|\omega|^{2 H+1}}, \quad c>0, \quad 0<H<1, \quad \omega \in R^{1} .
$$

In (1.1), the "spectral density" $\phi_{1}(\omega)$ must be understood in some specific sense (e.g. in the sense of time-scale analysis (Flandrin, 1989) or in a limiting sense (Solo, 1992)) since $B_{H}$ is a nonstationary process.

Anh et al. (1999a,b) introduced fractional Riesz-Bessel motion (fRBm), which is a Gaussian process with stationary increments and the spectral density of the increments is given by

$$
\phi_{2}(\omega)=\frac{\eta}{|\omega|^{2 \beta}\left(1+\omega^{2}\right)^{\alpha}}, \quad \omega \in R^{1},
$$

where $\eta>0,-\frac{1}{2}<\beta<\frac{1}{2}$ and $\alpha \geqslant 0$ are parameters. It is noted that model (1.2) is well defined and that the spectral density of the increments of $\mathrm{fBm}$ is given by (1.2) with $\alpha=0$.

The significance of fRBm (via Eq. (1.2)) is in its behaviour when $|\omega| \rightarrow \infty$. It is noted that the $\phi_{2}(\omega)$ of (1.2) is well defined as $|\omega| \rightarrow \infty$ due to the presence of the component $\left(1+\omega^{2}\right)^{-\alpha}, \alpha>0$, which is the Fourier transform of the Bessel potential. As a result, the covariances $R(t)$ of the increments of $\mathrm{fRBm}$ are strong for small $|t|$. That is, large (resp. small) values of the increments tend to be followed by large (resp. small) values with probability sufficiently close to one. This is the clustering phenomenon observed in stochastic finance (e.g. Shiryaev, 1999, p. 365). This phenomenon is referred to as (second-order) intermittency in the turbulence literature (e.g. Frisch, 1995). Summarising, the increments of fRBm display both LRD and intermittency, while those of fBm have LRD but no intermittency.

In this paper, we shall consider the case where the spectral density of nonstationary Gaussian processes with stationary increments is of a general and flexible form. The spectral density function of $\mathrm{fRBm}$ is thus a special case of this general form. A continuous version of the Gauss-Whittle objective function is proposed. Estimation procedures for the parameters involved in the spectral density function are then investigated. Both the consistency and the asymptotic normality of the estimators of the parameters are established. In addition, a real example is presented to demonstrate the applicability of the estimation procedures.

The organization of this paper is as follows. Section 2 proposes the estimation procedures and the corresponding asymptotic properties. A real example is given in Section 3. Mathematical proofs are given in the appendix.

\section{The estimation procedures}

Let $\left\{X(t): t \in R^{1}=(-\infty, \infty)\right\}$ be a zero-mean Gaussian process. Denote the increments of $X(t)$ by $\Delta X(t, \tau)=X(t+\tau)-X(t)$ for $t, \tau \in R^{1}$. The Gaussian process $X(t)$ 
is said to have second-order stationary increments if

$$
D\left(t, \tau_{1}, \tau_{2}\right)=E\left\{\left[\Delta X\left(t+s, \tau_{1}\right)\right]\left[\Delta X\left(s, \tau_{2}\right)\right]\right\}
$$

is independent of $s$ for all $s, t, \tau_{1}, \tau_{2} \in R^{1}$. The function $D\left(t, \tau_{1}, \tau_{2}\right)$ then has the spectral representation

$$
D\left(t, \tau_{1}, \tau_{2}\right)=\int_{-\infty}^{\infty} \mathrm{e}^{\mathrm{i} t \omega}\left(1-\mathrm{e}^{-\mathrm{i} \tau_{1} \omega}\right)\left(1-\mathrm{e}^{\mathrm{i} \tau_{2} \omega}\right) \frac{1+\omega^{2}}{\omega^{2}} F(\mathrm{~d} \omega),
$$

where $F(\mathrm{~d} \omega)$ is a nonnegative measure on $R^{1}$ such that $\int_{-\infty}^{\infty} F(\mathrm{~d} \omega)<\infty$ (e.g. Yaglom, 1986).

We shall assume that $F(\mathrm{~d} \omega)$ is absolutely continuous with derivative of the form

$$
f(\omega)=f(\omega, \mu)=\frac{\eta}{|\omega|^{2 \gamma}\left(1+\omega^{2}\right)^{\alpha}} \frac{\omega^{2}}{1+\omega^{2}} \pi(\omega ; \alpha, \gamma), \quad \omega \in R^{1},
$$

where $\mu=(\alpha, \gamma, \eta) \in \Omega=[0, \infty) \times\left(\frac{1}{2}, \frac{3}{2}\right) \times(0, \infty)$, and $\pi(\omega)=\pi(\omega ; \alpha, \gamma)$ is a positive and continuous function to be specified in Condition 2.1. When $\pi(\omega) \equiv 1$, model (2.2) corresponds to the fractional Riesz-Bessel motion case.

According to (2.1) and (2.2), we can define the spectral density of $X(t)$ by

$$
g(\omega)=g(\omega, \mu)=\frac{1+\omega^{2}}{\omega^{2}} f(\omega)=\frac{\eta}{|\omega|^{2 \gamma}\left(1+\omega^{2}\right)^{\alpha}} \pi(\omega), \quad \omega \in R^{1} .
$$

Let $\mu_{0}$ be the true value of $\mu$. We assume that $\mu_{0}$ is in the interior of $\Omega_{0}$, a compact subset of $\Omega$.

We now define the estimator of $g(\omega)$ by

$$
I_{N}^{X}(\omega)=\frac{1}{2 \pi N}\left|\int_{0}^{N} \mathrm{e}^{-\mathrm{i} \omega t} X(t) \mathrm{d} t\right|^{2},
$$

where $N>0$ is the upper bound of the interval $[0, N]$, on which each $X(t)$ is observed. Throughout this paper, the stochastic integrals are limits in mean square of appropriate Riemann sums. We assume that $X(0)=0$. It can be easily shown that the conclusions of Theorems 2.1 and 2.2 are not affected when $X(t)$ is replaced by $X_{-}(t)=X(t)-X(0)$ if $X(0) \neq 0$.

Note that the question of whether $I_{N}^{X}(\omega)$ is a consistent estimate of $g(\omega)$ has not been answered in the literature.

We need to introduce the following condition.

Condition 2.1. (i) Assume that $\pi(\omega ; \alpha, \gamma)$ is a positive and continuous function in both $\omega$ and $(\alpha, \gamma)$, bounded away from zero and chosen to satisfy

$$
\int_{-\infty}^{\infty} f(\omega, \mu) \mathrm{d} \omega<\infty \text { for } \mu \in \Omega .
$$

In addition, $\pi(\omega ; \alpha, \gamma)$ is a symmetric function in $\omega$ satisfying $0<\lim _{\omega \rightarrow 0} \pi(\omega ; \alpha, \gamma)<\infty$ and $0<\lim _{\omega \rightarrow \infty} \pi(\omega ; \alpha, \gamma)<\infty$ uniformly in $(\alpha, \gamma) \in[0, \infty) \times\left(\frac{1}{2}, \frac{3}{2}\right)$.

(ii) $\lim _{(\omega \rightarrow 0} \omega^{2} g\left(\omega, \mu_{0}\right)=0$. 
(iii) For $\varepsilon \leqslant\left\|\mu-\mu_{0}\right\|<\frac{1}{4}$ with any small $\varepsilon>0$,

$$
\int_{-\infty}^{\infty} \frac{g\left(\omega, \mu_{0}\right)}{g(\omega, \mu)} \frac{1}{1+\omega^{2}} \mathrm{~d} \omega<\infty .
$$

Now we state the following result and its proof is relegated to the appendix.

Theorem 2.1. Assume that Condition 2.1 holds. In addition, if

$$
\frac{1}{2}<\gamma<1 \text { and } \alpha \geqslant 0
$$

then we have as $N \rightarrow \infty$

$$
\begin{aligned}
& E\left[I_{N}^{X}(\omega)\right] \rightarrow g\left(\omega, \mu_{0}\right), \\
& E\left\{I_{N}^{X}(\omega)-E\left[I_{N}^{X}(\omega)\right]\right\}^{2}=g^{2}\left(\omega, \mu_{0}\right)+o(1)
\end{aligned}
$$

and

$$
\left.E\left\{\left(I_{N}^{X}(\omega)-E\left[I_{N}^{X}(\omega)\right]\right)\left(I_{N}^{X}(\lambda)\right)-E\left[I_{N}^{X}(\lambda)\right]\right)\right\}=\mathrm{o}(1)
$$

for all $\omega \neq 0, \lambda \neq 0$ and $\omega \neq \lambda$.

Remark 2.1. (i) The conditions of Theorem 2.1 are quite natural and mild. Condition 2.1(i) allows the dependence of $\pi(\omega)$ on $\alpha$ and $\gamma$ in model (2.2). This provides more flexible models than the following form:

$$
f(\omega)=f(\omega, \mu)=\frac{f^{*}(\omega)}{|\omega|^{2 \gamma}\left(1+\omega^{2}\right)^{\alpha}},
$$

where $f^{*}(\omega)$ is just a positive and continuous function of $\omega$. A similar model for time series with only long-range dependence has already been discussed. See model (1) of Hurvich et al. (1998). Both Conditions 2.1(ii) and 2.1(iii) hold automatically when $\pi(\omega)$ is just a function of $\omega$.

(ii) It is possible to further generalize model (2.2) to a form similar to model (1.4) of Robinson (1997). For this case, one needs to impose some more detailed conditions on $\pi(\omega)$. As the main objective of this paper is to estimate the parameters $\eta, \alpha$ and $\gamma$, we shall use only the decomposed form (2.2).

(iii) For the process $X(t)$, Theorem 2.1 shows that $I_{N}^{X}(\omega)$ is asymptotically unbiased while $I_{N}^{X}(\omega)$ is not a weakly consistent estimator of $g(\omega)$. In addition, Eq. (2.4) justifies the fact that $g(\omega)$ can be defined as a spectral density of $X(t)$. Recently, Solo (1992) established (2.4) for the case where

$$
g(\omega)=|\omega|^{-2 i} \quad \text { for } \frac{1}{2}<\gamma \leqslant 1 .
$$

This part of the paper considers estimating the parameters involved in the spectral density of the nonstationary process $X(t)$. For this case, we define the following 
continuous version of the Gauss-Whittle objective function:

$$
L_{N}^{X}(\mu)=\frac{1}{4 \pi} \int_{-\infty}^{\infty}\left\{\log (g(\omega, \mu))+\frac{I_{N}^{X}(\omega)}{g(\omega, \mu)}\right\} \frac{\mathrm{d} \omega}{1+\omega^{2}}
$$

and the minimum contrast estimator of $\mu$ by

$$
\hat{\mu}_{N}=\arg \min _{\mu \in \Omega_{0}} L_{N}^{X}(\mu)
$$

where $\Omega_{0}$ is a compact subset of $\Omega$.

Remark 2.2. This paper considers using the continuous version of the Gauss-Whittle contrast function to estimate the spectral density $g(\omega)$ with its frequency defined on $(-\infty, \infty)$. For the continuous case, due to the slow decay of the spectral density at $\infty$, the weight function $1 /\left(1+\omega^{2}\right)$ must be used in (2.7) to ensure that Eq. (2.7) is well defined. Eq. (2.7) can also be justified by applying the entropy theory discussed in Dym and McKean (1976).

The weak consistency is given below and its proof is postponed to the appendix.

Theorem 2.2. Assume that the conditions of Theorem 2.1 hold. In addition, if the true value parameter $\mu_{0}$ of $\mu$ is in the interior of $\Omega_{0}$, then

$$
\hat{\mu}_{N} \rightarrow \mathrm{p} \mu_{0} \text { as } N \rightarrow \infty .
$$

Remark 2.3. It is worthwhile to point out that condition (2.3) covers the important case where $\frac{1}{2}<\gamma<1$ and $0<\alpha<1$. More recently, Gao et al. (2001) consider another important case where $X(t)$ is a stationary Gaussian process and the parameters involved in its spectral density $g(\omega)$ satisfy $0<\gamma<\frac{1}{2}$ and $\alpha \geqslant \frac{1}{2}$. The latter case allows a stationary process to display both LRD and second-order intermittency.

As pointed out in Anh et al. (1999a,b), it is more useful to establish a consistent estimator for $\mu$ for the case where

$$
1 \leqslant \gamma<\frac{3}{2} \text { and } \alpha \geqslant 0 .
$$

Due to the fact that (2.4) and (2.5) are only true under condition (2.3), we can only establish a weakly consistent estimator for $\mu$ under condition (2.3). Therefore, in order to construct a consistent estimation procedure for the case of (2.8), we need to transform the nonstationary process $X(t)$ into a stationary process. This paper suggests using the following transform.

Define the Haar wavelet function

$$
h(u)= \begin{cases}1, & 0 \leqslant u<\frac{1}{2}, \\ -1, & \frac{1}{2} \leqslant u<1, \\ 0, & \text { otherwise }\end{cases}
$$


We have its Fourier transform $\hat{\psi}$ given by

$$
\hat{h}(t)=\frac{\mathrm{i} t}{4} \mathrm{e}^{-\mathrm{i} t / 2}\left(\frac{\sin (t / 4)}{t / 4}\right)^{2} \text {. }
$$

We now define a new process

$$
Y(t)=\int_{-\infty}^{\infty} X(s) h(s-t) \mathrm{d} s=\left\{\int_{0}^{1 / 2} X(t+u) \mathrm{d} u-\int_{1 / 2}^{1} X(t+u) \mathrm{d} u\right\}, \quad t \in R^{1} .
$$

Then, it follows from (2.1) and (2.2) that

$$
\begin{aligned}
E[Y(t+\tau) Y(t)] & \int_{-\infty}^{\infty} \int_{-\infty}^{\infty} E[X(u+t+\tau) X(v+t)] h(u) h(v) \mathrm{d} u \mathrm{~d} v \\
= & \int_{-\infty}^{\infty} \int_{-\infty}^{\infty}\left(\int_{-\infty}^{\infty}\left(1-\mathrm{e}^{-\mathrm{i}(u+\tau) \omega}\right)\left(1-\mathrm{e}^{\mathrm{i} v \omega}\right) \frac{1+\omega^{2}}{\omega^{2}} f(\omega) \mathrm{d} \omega\right) h(u) h(v) \mathrm{d} u \mathrm{~d} v \\
= & \int_{-\infty}^{\infty}\left(\int_{-\infty}^{\infty}\left(1-\mathrm{e}^{-\mathrm{i}(u+\tau) \omega}\right) h(u) \mathrm{d} u\right. \\
& \left.\int_{-\infty}^{\infty}\left(1-\mathrm{e}^{\mathrm{i} v \omega}\right) h(v) \mathrm{d} v\right) \frac{1+\omega^{2}}{\omega^{2}} f(\omega) \mathrm{d} \omega \\
= & \int_{-\infty}^{\infty} \mathrm{e}^{-\mathrm{i} \tau x}|\hat{h}(\omega)|^{2} \frac{1+\omega^{2}}{\omega^{2}} f(\omega) \mathrm{d} \omega,
\end{aligned}
$$

which is independent of $t$. Consequently, the process $Y(t)$ is a stationary Gaussian process with the following spectral density:

$$
\phi(\omega)=\phi(\omega, \theta)=|\hat{h}(\omega)|^{2} \frac{1+\omega^{2}}{\omega^{2}} f(\omega)=\frac{1}{16} \frac{\eta}{|\omega|^{2 \beta}} \frac{\pi(\omega ; \alpha, \beta)}{\left(1+\omega^{2}\right)^{\alpha}}\left(\frac{\sin (\omega / 4)}{\omega / 4}\right)^{4},
$$

where $\omega \in R^{1}, \beta=\gamma-1$ and $\theta=(\alpha, \beta, \eta) \in \Theta=[0, \infty) \times[0,1 / 2) \times(0, \infty)$. For convenience, we use $\pi(\omega)=\pi(\omega ; \alpha, \beta)=\pi(\omega ; \alpha, \gamma)$ throughout the rest of the paper. Let $\theta_{0}$ be the true value of $\theta$. We assume that $\theta_{0}$ is in the interior of $\Theta_{0}$, a compact subset of $\Theta$.

Remark 2.4. (i) We believe that both the wavelet transform and the resulting estimation procedure provide a general approach to the estimation of (locally) self-similar processes. In addition, as pointed out in Vergassola and Frish (1991), the wavelet transform is continuous and linear, and the process $Y(t)$ is itself Gaussian when the process $X(t)$ is Gaussian. By contrast, the usual increments do not necessarily retain the Gaussianity property.

(ii) We also need to point out that this paper adopts a simple form of the Haar wavelet function for the stationarity transform. When using a more complicated form, the structure of $f(\omega)$ will remain unchanged. The only change is the form of $\hat{h}(\cdot)$, which will not affect the estimation of the parameters. See Anh et al. (1999a,b). In 
addition, our experience suggests that the choice of the simple form of $h(\cdot)$ can provide stable simulation and numerical results.

(iii) The main assumption of this paper is that the nonstationary process $X(t)$ is Gaussian. If $X(t)$ is not Gaussian but the increments of $X(t)$ are assumed to be Gaussian, we can still estimate the parameter $\theta$. One can use for example the first difference $Y(t)=X(t+1)-X(t)$ to obtain a stationary process. If $Y(t)$ is Gaussian, then its spectral density can be proved to be

$$
\phi_{Y}(\omega)=\frac{\eta}{|\omega|^{2 \beta}} \frac{\pi(\omega ; \alpha, \beta)}{\left(1+\omega^{2}\right)^{\alpha}}\left(\frac{\sin (\omega / 2)}{\omega / 2}\right)^{2},
$$

where $\theta$ is as defined above. It can be seen that the conclusions of Theorems 2.4 and 2.5 below remain true.

For any given $\omega \in(-\infty, \infty)$, we define the estimator of $\phi(\omega)=\phi(\omega, \theta)$ by

$$
I_{N}^{Y}(\omega)=\frac{1}{2 \pi N}\left|\int_{0}^{N} \mathrm{e}^{-\mathrm{i} \omega t} Y(t) \mathrm{d} t\right|^{2} .
$$

For the periodogram $I_{N}^{Y}(\omega)$, we have the following consistency result and its proof is postponed to the appendix.

Theorem 2.3. Assume that condition (2.8) holds. In addition, $Y(0)=0$. Then we have as $N \rightarrow \infty$

$$
\begin{aligned}
& E\left[I_{N}^{Y}(\omega)\right]-\phi\left(\omega, \theta_{0}\right)=\mathrm{O}(1 / N), \\
& \left.E\left\{I_{N}^{Y}(\omega)\right)-E\left[I_{N}^{Y}(\omega)\right]\right\}^{2}=\phi^{2}\left(\omega, \theta_{0}\right)+\mathrm{O}\left(1 / N^{2}\right)
\end{aligned}
$$

and

$$
\left.\left.E\left\{\left(I_{N}^{Y}(\omega)\right)-E\left[I_{N}^{Y}(\omega)\right]\right)\left(I_{N}^{Y}(\lambda)\right)-E\left[I_{N}^{Y}(\lambda)\right]\right)\right\}=\mathrm{O}\left(1 / N^{2}\right)
$$

for all $\omega \neq 0, \lambda \neq 0$ and $\omega \neq \lambda$.

Remark 2.5. Eq. (2.9) implies that $I_{N}^{Y}(\omega)$ is asymptotically unbiased while (2.10) shows that $I_{N}^{Y}(\omega)$ is not a consistent estimator of $\phi(\omega)$, although $Y(t)$ is now stationary. Results (2.9)-(2.11) are similar to those for the discrete time processes with short-range dependence. See for example, Chapter 10 of Brockwell and Davis (1990).

As mentioned earlier, the main objective of this paper concentrates on the estimation of $\theta$. This paper then considers using an extended version of the Gauss-Whittle contrast function to estimate the spectral density $\phi(\omega)$ with its frequency defined on $(-\infty, \infty)$. When considering the case where the spectral density is defined on $(-\pi, \pi]$, the weight function $1 /\left(1+\omega^{2}\right)$ is not required (see, for example, Heyde and Gay, 1993 for example).

For the stationary process $Y(t)$, we define the following objective function:

$$
L_{N}^{Y}(\theta)=\frac{1}{4 \pi} \int_{-\infty}^{\infty}\left\{\log (\phi(\omega, \theta))+\frac{I_{N}^{Y}(\omega)}{\phi(\omega, \theta)}\right\} \frac{\mathrm{d} \omega}{1+\omega^{2}} .
$$


The minimum contrast estimator of $\theta$ is defined by

$$
\hat{\theta}_{N}=\arg \min _{\theta \in \Theta_{0}} L_{N}^{Y}(\theta)
$$

where $\Theta_{0}$ is a compact subset of $\Theta$.

In order to state the asymptotic normality of $\hat{\theta}_{N}$, we need to introduce the following conditions:

Condition 2.2. (i) Assume that the first two derivatives of $\log (\phi(\omega, \theta))$ in $\theta$ exist and are continuous in $\omega \in(-\infty, \infty)$ and $\theta \in \Theta$.

(ii) Assume that

$$
\left.\int_{-\infty}^{\infty} \frac{\phi^{2}\left(\omega, \theta_{0}\right)}{\left(1+\omega^{2}\right)^{2}}\left(\frac{\partial \log (\phi(\omega, \theta))}{\partial \theta}\right)^{T}\left(\frac{\partial \log (\phi(\omega, \theta))}{\partial \theta}\right)\right|_{\theta=\theta_{0}}<\infty
$$

where

$$
\frac{\partial \log (\phi(\omega, \theta))}{\partial \theta}=\left(\frac{\partial \log (\phi(\omega, \theta))}{\partial \alpha}, \frac{\partial \log (\phi(\omega, \theta))}{\partial \beta}, \frac{\partial \log (\phi(\omega, \theta))}{\partial \eta}\right)^{\tau} .
$$

(iii) For any real function $l \in L^{2}(-\infty, \infty)$,

$$
\left.\int_{-\infty}^{\infty} \frac{\phi^{2}\left(\omega, \theta_{0}\right)}{\left(1+\omega^{2}\right)^{2}} l^{2}(\omega)\left(\frac{\partial \log (\phi(\omega, \theta))}{\partial \theta}\right)^{T}\left(\frac{\partial \log (\phi(\omega, \theta))}{\partial \theta}\right)\right|_{\theta=\theta_{0}}<\infty .
$$

(iv) Assume that $\sum_{k=-\infty}^{\infty} \phi(\omega-2 k \pi, \theta)$ converges uniformly in $\omega \in(-\pi, \pi] \backslash\{0\}$ and $\theta \in \Theta_{0}$.

Condition 2.3. (i) For $\theta \in \Theta$,

$$
\Gamma(\theta)=\frac{1}{4 \pi} \int_{-\infty}^{\infty}\left(\frac{\partial \log (\phi(\omega, \theta))}{\partial \theta}\right)\left(\frac{\partial \log (\phi(\omega, \theta))}{\partial \theta}\right)^{T} \frac{1}{\left(1+\omega^{2}\right)^{2}} \mathrm{~d} \omega<\infty .
$$

(ii) The inverse matrix, $\Gamma^{-1}\left(\theta_{0}\right)$, of $\Gamma\left(\theta_{0}\right)$ does exist.

Remark 2.6. (i) Conditions 2.2 and 2.3 are similar to those for the discrete case. See for example, Condition (A2) of Heyde and Gay (1993).

(ii) Conditions 2.2 and 2.3 indirectly impose some restrictions on the form of $\pi(\omega)$. When $\pi(\omega) \equiv 1$, Conditions 2.2 and 2.3 hold automatically. The justification is similar to that of Lemma B.1 of Gao et al. (2001).

(iii) Condition 2.2(iii) is required for an application of a continuous-time central limit theorem to the proof of the asymptotic normality.

We now state the next result of this paper and its proof is postponed to the appendix.

Theorem 2.4. (i) Assume that Condition 2.1 holds. In addition, if the true value $\theta_{0}$ of $\theta$ is in the interior of $\Theta_{0}$, then under condition (2.8)

$$
\hat{\theta}_{N} \rightarrow \theta_{0} \quad \text { with probability one }
$$

as $N \rightarrow \infty$. 
(ii) Assume that Conditions 2.1-2.3 hold. In addition, if the true value $\theta_{0}$ of $\theta$ is in the interior of $\Theta_{0}$, then under condition (2.8)

$$
\sqrt{N}\left(\hat{\theta}_{N}-\theta_{0}\right) \rightarrow_{\mathrm{D}} \mathrm{N}\left(0, \Gamma^{-1}\left(\theta_{0}\right)\right),
$$

where $\Gamma^{-1}\left(\theta_{0}\right)$ is as defined in Condition 2.3(ii).

Remark 2.7. (i) Theorem 2.4 establishes both the strong consistency and the asymptotic normality of $\hat{\theta}_{N}$. Previously, we were unable to establish the asymptotic normality of $\hat{\theta}_{N}$. Instead, we established the asymptotic normality for a discrete approximation of $\hat{\theta}_{N}$. See for example, Theorem 2.2 of Gao et al. (2001). Similarly, we can now establish the asymptotic normality of $\theta_{N}$ of Gao et al. (2001). Thus, for both the nonstationary and stationary cases, we can establish an asymptotically normal estimator for the vector of parameters involved in the spectral density. This theory is now complete.

(ii) Theorem 2.4 extends and complements some existing results. For example, Dahlhaus (1989) established asymptotic normality for estimators based the Whittle approach for the discrete case. Giraitis and Leipus (1995) discussed asymptotic consistency for estimators based the Whittle approach for the discrete case. Viano et al. (1994) established some probabilistic and asymptotic results for continuous-time fractional ARMA processes. Viano et al. (1995) considered a class of extended fractional ARMA processes, and discussed the asymptotic behaviour of their correlations.

As $Y(t)$ is stationary and Gaussian, we now can study the asymptotic distribution of $I_{N}^{Y}(\omega)$ for any fixed $\omega$. Similar results for the discrete case have been discussed extensively (see, for example, Künsch, 1986; Hurvich and Beltrao, 1993; Robinson, 1995).

We state the last result of this section and its proof is postponed to the appendix.

Theorem 2.5. Assume that the conditions of Theorem 2.3 hold. Then the normalized periodogram $I_{N}^{Y}(\omega) / \phi\left(\omega, \theta_{0}\right)$ is asymptotically distributed as $\frac{1}{2} \chi_{2}^{2}$.

Remark 2.8. For the discrete case, Theorem 6 of Hurvich and Beltrao (1993) shows that $I_{N}^{Y}(\omega) / \phi\left(\omega, \theta_{0}\right)$ at a given frequency is asymptotically distributed as a quadratic form, and is only asymptotically distributed as $\frac{1}{2} \chi_{2}^{2}$ for a specific case. By contrast, Theorem 2.5 shows that for the continuous case the asymptotic distribution of $I_{N}^{Y}(\omega)$ / $\phi\left(\omega, \theta_{0}\right)$ for any fixed $\omega$ is $\frac{1}{2} \chi_{2}^{2}$.

\section{Implementation and application}

In many practical circumstances, however, observations on $Y(t)$ are made at discrete intervals of time, even though the underlying process may be continuous. In addition, it is computationally easier to find a consistent estimate of $\theta$ based on a sequence of discrete observations on $Y(t)$. This section considers the following discrete 
process:

$$
Z_{t}=Y(t), \quad t=1,2, \ldots
$$

According to (3.1), the autocovariance function of $Z_{t}$ is well defined by

$$
r_{\tau}=\operatorname{cov}\left(Z_{t}, Z_{t+\tau}\right)=\operatorname{cov}\{Y(t), Y(t+\tau)\}=\int_{-\infty}^{\infty} \mathrm{e}^{\mathrm{i} \tau \omega} \phi(\omega, \theta) \mathrm{d} \omega
$$

since $\int_{-\infty}^{\infty}|\phi(\omega, \theta)| \mathrm{d} \omega<\infty$.

Then it can be shown that

$$
\begin{aligned}
r_{\tau} & =\int_{-\infty}^{\infty} \mathrm{e}^{\mathrm{i} \tau(\omega)} \phi(\omega, \theta) \mathrm{d} \omega=\sum_{k=-\infty}^{\infty} \int_{(2 k-1) \pi}^{(2 k+1) \pi} \mathrm{e}^{\mathrm{i} \tau \omega} \phi(\omega, \theta) \mathrm{d} \omega \\
& =\sum_{k=-\infty}^{\infty} \int_{-\pi}^{\pi} \exp \{\mathrm{i} \tau(\omega-2 k \pi)\} \phi(\omega-2 k \pi, \theta) \mathrm{d} \omega \\
& =\int_{-\pi}^{\pi} \exp (\mathrm{i} \tau \omega)\left\{\sum_{k=-\infty}^{\infty} \phi(\omega-2 k \pi, \theta)\right\} \mathrm{d} \omega
\end{aligned}
$$

since $\sum_{k=-\infty}^{\infty} \phi(\omega-2 k \pi, \theta)$ converges uniformly in $\omega \in(-\pi, \pi] \backslash\{0\}$ and $\theta \in \Theta_{0}$.

Therefore, the spectral density function of $Z_{t}$ can be defined by

$$
f_{Z}(\omega)=f(\omega, \theta)=\sum_{k=-\infty}^{\infty} \phi(\omega-2 k \pi, \theta)
$$

We now define the following discrete version of the Gauss-Whittle contrast function:

$$
W(\theta)=W_{T}(\theta)=\frac{1}{2 T} \sum_{s=1}^{T-1}\left\{\log \left(f\left(\omega_{s}, \theta\right)\right)+\frac{I_{T}^{Z}\left(\omega_{s}\right)}{f\left(\omega_{s}, \theta\right)}\right\},
$$

where $T=[N] \leqslant N$ is the smallest integer part, $\omega_{s}=2 \pi s / T$, and

$$
I_{T}^{Z}(\omega)=\frac{1}{2 \pi T}\left|\sum_{t=1}^{T} \mathrm{e}^{\mathrm{i} \omega t} Z_{t}\right|^{2}
$$

Now, the minimum contrast estimator of $\theta$ can be defined by

$$
\tilde{\theta}_{T}=\arg \min _{\theta \in \Theta_{0}} W(\theta)
$$

It can be shown that as $N \rightarrow \infty$

$$
\tilde{\theta}_{T}-\hat{\theta}_{N} \rightarrow \mathrm{p} 0
$$

The derivation of (3.2)-(3.4) is similar to (2.4)-(2.6) of Gao et al. (2001). 
Assume that the solution of (3.3) satisfies

$$
W^{\prime}\left(\tilde{\theta}_{T}\right)=0
$$

Similar to Appendix C of Gao et al. (2001), one can implement (3.5) in practice.

Example 3.1. In this example, we consider two air pollution data sets provided by the Environment Protection Authority of New South Wales (NSWEPA) of Australia: the Lidcombe $N O_{x}$ time series (1) and the Lidcombe ozone time series (2). These are measurements of maximum daily concentrations and cover the period 2/1/1982$30 / 4 / 1994$. Lidcombe is an urban suburb at the centre of Sydney. Lidcombe is chosen because, at this site of the NSWEPA monitoring network, long series of monitoring data are available in a fairly complete form. We apply our estimation procedure to determine the values of the parameters and to detect whether the data sets exhibit both long-range dependence and intermittency. If we assume that the two data sets are stationary and Gaussian, then the spectral density of the data set ( $i$ ) can be defined by

$$
\phi_{i}(\omega, \theta)=\frac{\eta_{i}}{|\omega|^{2 \beta_{i}}\left(1+\omega^{2}\right)^{\alpha_{i}}}\left(\frac{\sin (\omega / 4)}{\omega / 4}\right)^{4}, \quad \omega \in(-\infty, \infty),
$$

where $\alpha_{i} \in[0, \infty), \beta_{i} \in(-1 / 2,1 / 2)$ and $0<\eta_{i}<\infty$ for $i=1,2$. Note that the form of $\phi_{i}(\omega, \theta)$ is the special case of $\phi(\omega, \theta)$ where $\pi(\omega) \equiv 16$.

The plots and the power spectra of the data sets are given in Figs. 1-4. We should note the singularity at frequency 0 and the power law form of the sample spectra, indicating the presence of long-range dependence in the data. Applying the estimation procedure presented in Section 2 and the computational formulae given in (3.1)-(3.5) to the data sets, we found the solution of (3.5), and the values of the unknown parameters in (3.6) were $\alpha_{1}=0.04474, \beta_{1}=0.2668, \eta_{1}=12.571, \alpha_{2}=0.07832, \beta_{2}=0.3396$ and $\eta_{2}=359.618$, respectively. These results confirm that the two data sets exhibit both long-range dependence $\left(\beta_{i}>0\right)$ and intermittency $\left(\alpha_{i}>0\right)$, although the intermittency is relatively less pronounced.

Remark 3.1. This example has described some basic features of the air pollution time series for a single monitoring station at Lidcombe. The identification of long-range dependence and intermittency of the time series has facilitated the use of modern regression analysis to model the dependence of the maximum daily ozone on some factors such as morning temperature and wind speed. Given the complexity of air pollution time series, we suggest using nonparametric time series regression smoothing techniques (e.g. Fan and Gijbels, 1996) or semiparametric time series regression methods (e.g. Härdle et al., 2000) to model the dependence and then to provide better forecasts.

Another issue is the selection of avenues, as there are many avenues for further investigations. The characteristics of air quality at the other sites of Sydney could be investigated in a similar manner. These characteristics can be used to identify the structural relationships between different monitoring stations and different subregions 


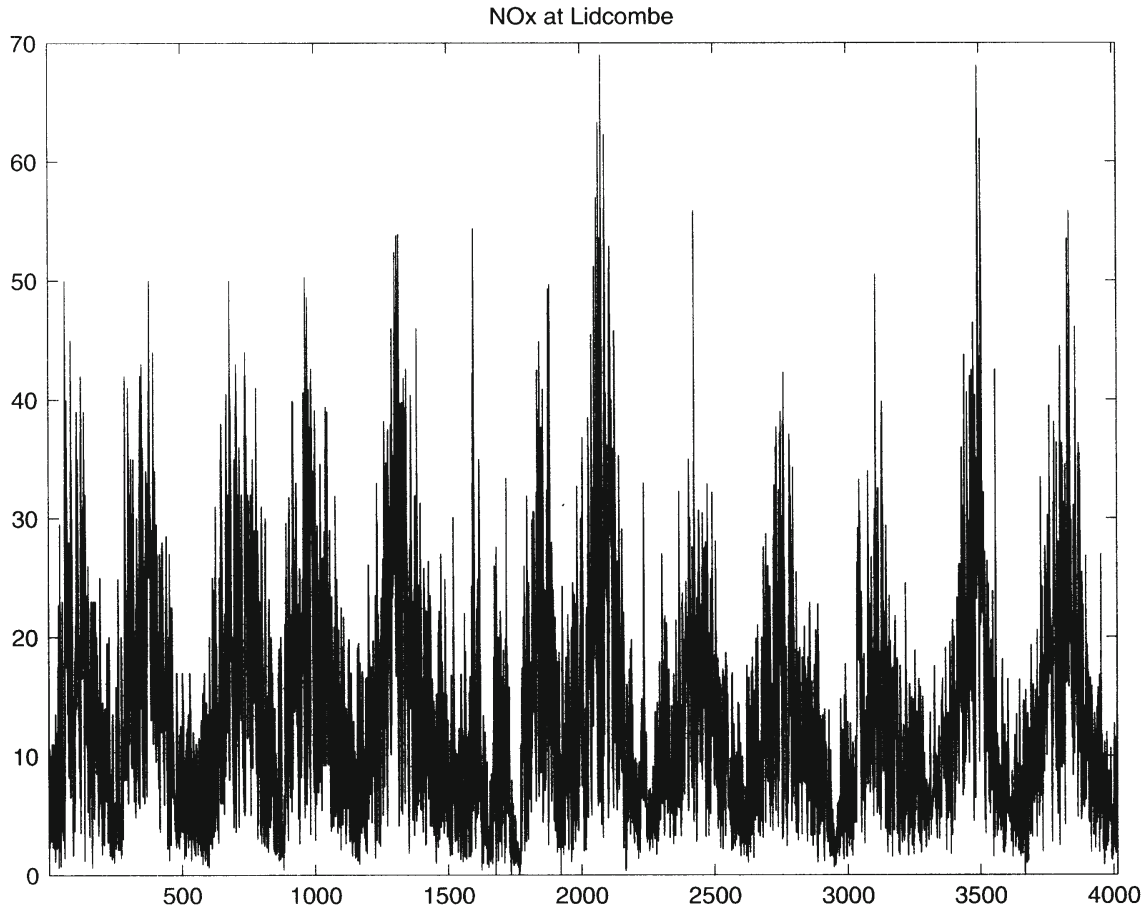

Fig. 1 .

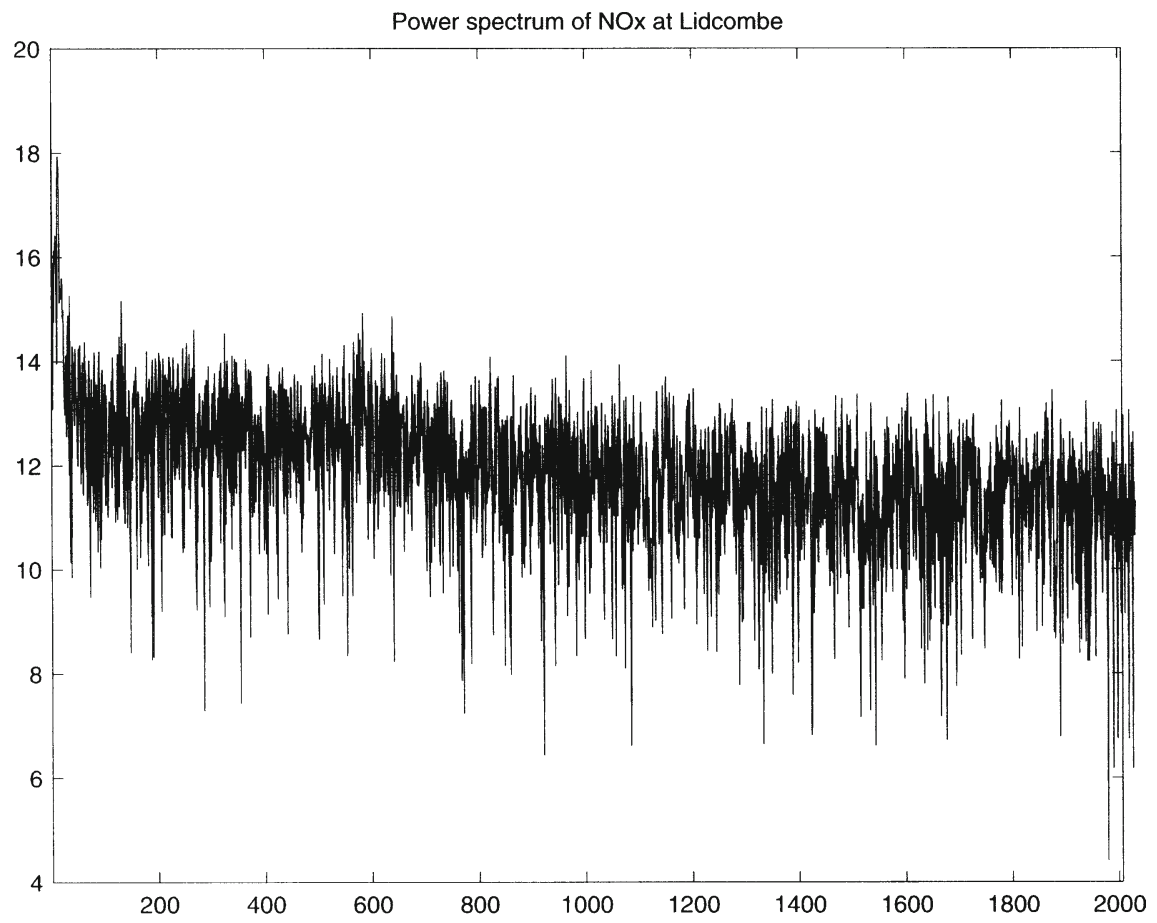

Fig. 2 . 


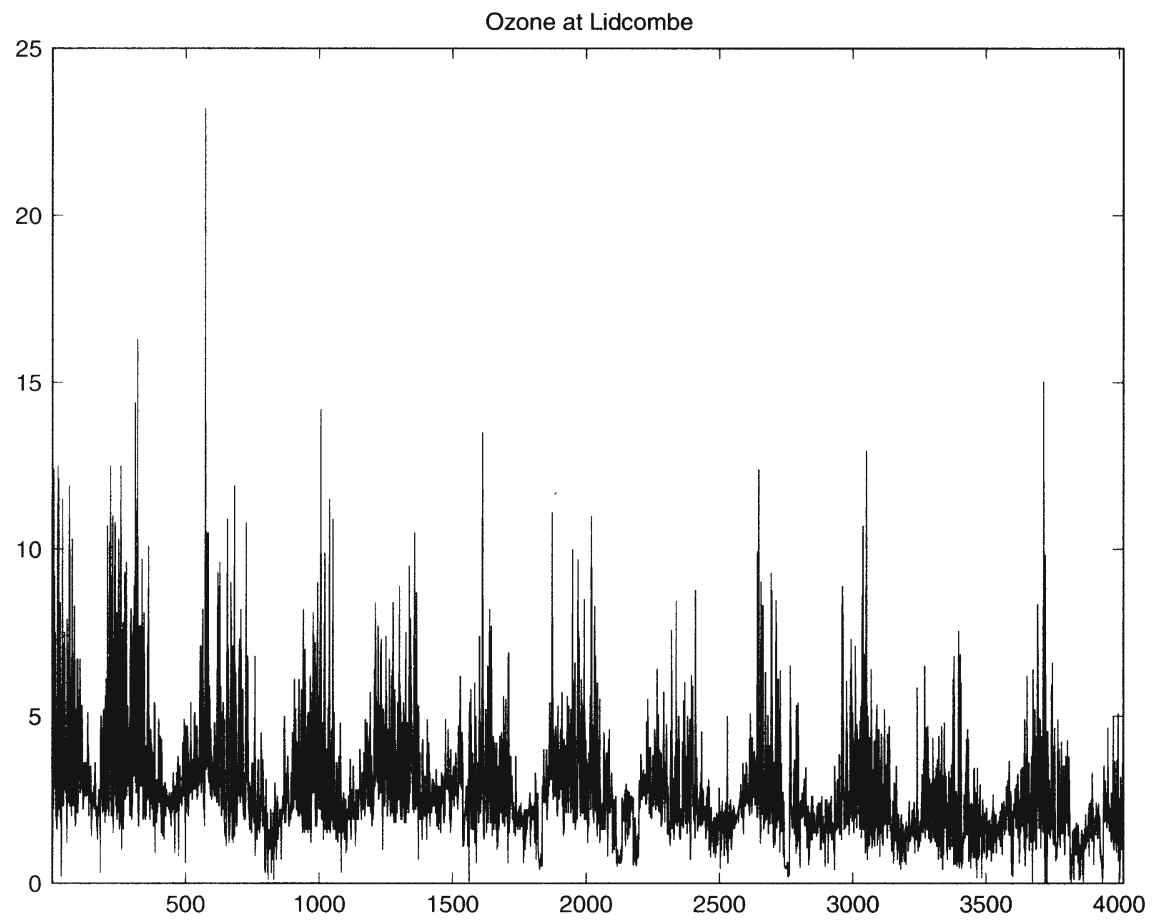

Fig. 3.

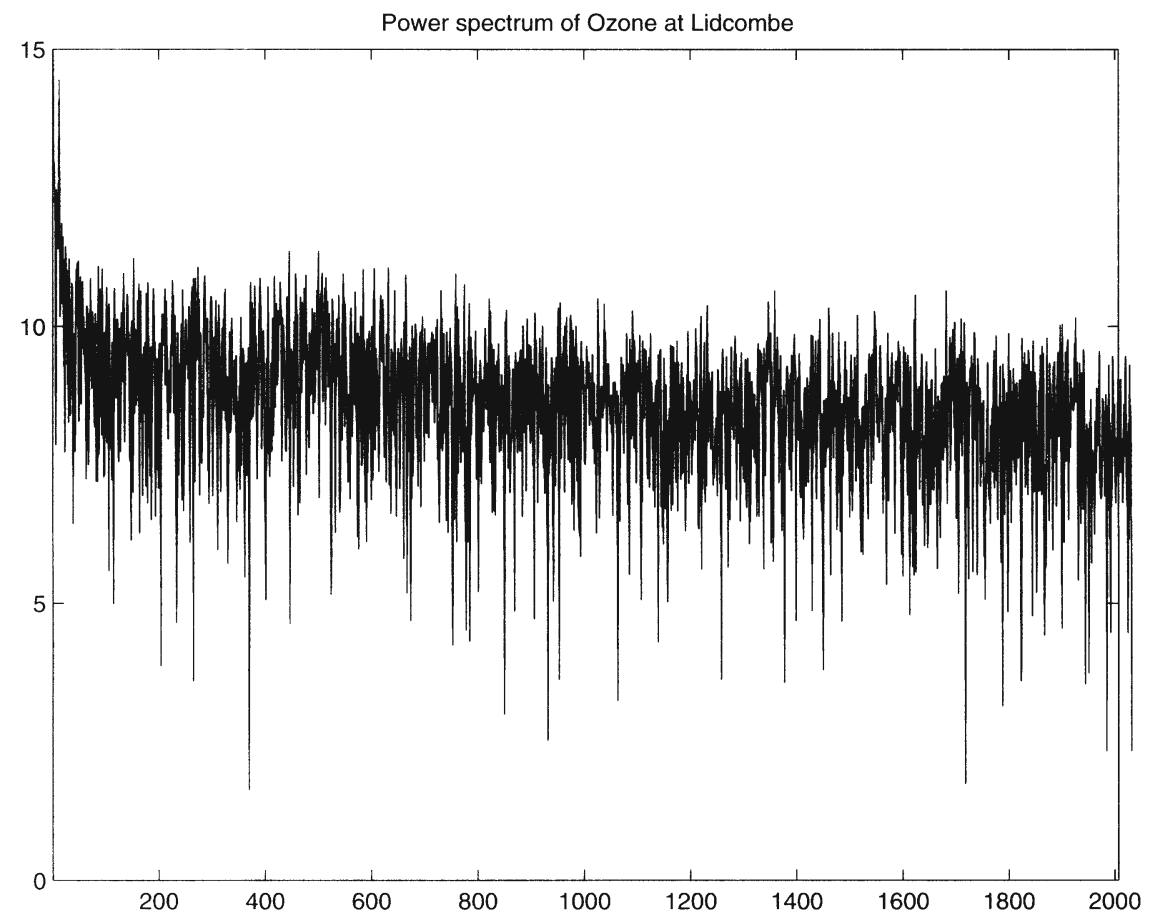

Fig. 4 . 
of the Sydney airshed. For a more reliable forecasting system, it is worthwhile to investigate a much fuller set of information over all subregions.

\section{Acknowledgements}

The authors would like to thank the Editor, the Associate Editor and the referees for their constructive comments and suggestions. Thanks also go to the Australian Research Council for its financial support.

\section{Appendix A}

Proof of Theorem 2.1. It now follows from (2.1) and (2.2) that

$$
E[X(s) X(t)]=\int_{-\infty}^{\infty}\left(1-\mathrm{e}^{\mathrm{i} t \lambda}\right)\left(1-\mathrm{e}^{-\mathrm{i} s \lambda}\right) g_{0}(\lambda) \mathrm{d} \lambda .
$$

Thus

$$
\begin{aligned}
E\left[I_{N}^{X}(\omega)\right] & =\frac{1}{2 \pi N} \int_{-\infty}^{\infty}\left(\int_{0}^{N} \int_{0}^{N}\left(1-\mathrm{e}^{\mathrm{i} t \lambda}\right) \mathrm{e}^{-\mathrm{i} \omega t}\left(1-\mathrm{e}^{-\mathrm{i} s \lambda}\right) \mathrm{e}^{\mathrm{i} \omega s} \mathrm{~d} s \mathrm{~d} t\right) g_{0}(\lambda) \mathrm{d} \lambda \\
& =\frac{N}{2 \pi} \int_{-\infty}^{\infty}|B(\omega N)-B((\omega-\lambda) N)|^{2} g_{0}(\lambda) \mathrm{d} \lambda
\end{aligned}
$$

where $B(x)=\left(\mathrm{e}^{\mathrm{i} x}-1\right) / \mathrm{ix}$ and $g_{0}(\lambda)=g\left(\lambda, \mu_{0}\right)$.

Next, using the identity

$$
B(\omega N)-B((\omega-\lambda) N)=\lambda\left(B((\omega-\lambda) N)-\mathrm{e}^{\mathrm{i} \omega N} B(\omega N)\right) / \omega
$$

we have

$$
\begin{aligned}
E\left[I_{N}^{X}(\omega)\right]= & \frac{N}{2 \pi \omega^{2}} \int_{-\infty}^{\infty} \psi((\omega-\lambda) N)^{2} g_{0}(\lambda) \lambda^{2} \mathrm{~d} \lambda \\
& +\frac{N}{2 \pi \omega^{2}} \int_{-\infty}^{\infty} \psi(\lambda N)^{2} \lambda^{2} g_{0}(\lambda) \mathrm{d} \lambda \\
& -\frac{2 N}{2 \pi \omega^{2}} \int_{-\infty}^{\infty} \psi((\omega-\lambda) N) \psi(\lambda N) \lambda^{2} g_{0}(\lambda) \mathrm{d} \lambda \equiv E_{1 N}+E_{2 N}-E_{12 N},
\end{aligned}
$$

where $\psi(\omega)=\sin (\omega / 2) /(\omega / 2)$, the symbol " $\equiv$ " indicates that the terms on the left-hand side are represented correspondingly by those on the right-hand side. 
Now, by Fejer's theorem (e.g. Goldberg, 1961, p. 17), we have as $N \rightarrow \infty$

$$
E_{1 N} \rightarrow g_{0}(\omega)=g\left(\omega, \mu_{0}\right), \quad E_{2 N} \rightarrow \frac{1}{\omega^{2}} \lim _{\lambda \rightarrow 0}\left(g_{0}(\lambda) \lambda^{2}\right)=0
$$

and

$$
\frac{\omega^{2}}{2 \psi(\omega N)} E_{12 N} \rightarrow \lim _{\lambda \rightarrow 0}\left(g_{0}(\lambda) \lambda^{2}\right)=0
$$

using Condition 2.1(ii).

Therefore, the proof of (2.3) is completed.

Before proving (2.5), we introduce the following notation and identity:

$$
\begin{aligned}
& r_{X}(s, t, u, v)=E[X(s) X(t) X(u) X(v)] \quad \text { and } \quad r_{X}(s, t)=E[X(s) X(t)], \\
& r_{X}(s, t, u, v)-r_{X}(s, t) r_{X}(u, v)=r_{X}(s, u) r_{X}(t, v)+r_{X}(s, v) r_{X}(t, u)
\end{aligned}
$$

since $X(t)$ is Gaussian and $X(0)=0$.

It follows from (2.1) and (2.2) again that

$$
\begin{aligned}
4 & \pi^{2} N^{2} E\left\{I_{N}^{X}(\omega)-E\left[I_{N}^{X}(\omega)\right]\right\}^{2} \\
= & \int_{0}^{N} \int_{0}^{N} \int_{0}^{N} \int_{0}^{N}\left\{\left[r_{X}(s, t, u, v)-r_{X}(s, t) r_{X}(u, v)\right]\right\} \mathrm{e}^{\mathrm{i} \omega(t-s)+\mathrm{i} \omega(u-v)} \mathrm{d} s \mathrm{~d} t \mathrm{~d} u \mathrm{~d} v \\
= & \int_{0}^{N} \int_{0}^{N} \int_{0}^{N} \int_{0}^{N} r_{X}(s, v) r_{X}(t, u) \mathrm{e}^{\mathrm{i} \omega(t+u)-\mathrm{i} \omega(s+v)} \mathrm{d} t \mathrm{~d} u \mathrm{~d} s \mathrm{~d} v \\
& +\int_{0}^{N} \int_{0}^{N} \int_{0}^{N} \int_{0}^{N} r_{X}(s, u) r_{X}(t, v) \mathrm{e}^{\mathrm{i} \omega(t-v)+\mathrm{i} \omega(u-s)} \mathrm{d} t \mathrm{~d} v \mathrm{~d} s \mathrm{~d} u .
\end{aligned}
$$

Similar to (A.1) and (A.2), we have

$$
\begin{aligned}
\frac{1}{N} \int_{0}^{N} \int_{0}^{N} r_{X}(t, u) \mathrm{e}^{\mathrm{i} \omega(t+u)} \mathrm{d} t \mathrm{~d} u \\
=-\frac{N}{\omega^{2}} \int_{-\infty}^{\infty}\left[B((\lambda+\omega) N)+\mathrm{e}^{\mathrm{i} \omega N} B(\lambda N)\right] \\
\quad \times\left[B((\omega-\lambda) N)+\mathrm{e}^{\mathrm{i} \omega N} B(-\lambda N)\right] \lambda^{2} g_{0}(\lambda) \mathrm{d} \lambda
\end{aligned}
$$

and

$$
\begin{aligned}
\frac{1}{N} \int_{0}^{N} \int_{0}^{N} r_{X}(s, v) \mathrm{e}^{-\mathrm{i} \omega(s+v)} \mathrm{d} s \mathrm{~d} v \\
=-\frac{N}{\omega^{2}} \int_{-\infty}^{\infty}\left[B((\lambda-\omega) N)+\mathrm{e}^{-\mathrm{i} \omega N} B(\lambda N)\right] \\
\quad \times\left[B(-(\lambda+\omega) N)+\mathrm{e}^{-\mathrm{i} \omega N} B(-\lambda N)\right] \lambda^{2} g_{0}(\lambda) \mathrm{d} \lambda
\end{aligned}
$$


Observe that as $N \rightarrow \infty$

$$
\begin{aligned}
& \frac{N}{\omega^{2}} \int_{-\infty}^{\infty} B((\lambda+\omega) N) B((\omega-\lambda) N) \lambda^{2} g_{0}(\lambda) \mathrm{d} \lambda \\
& \quad=\frac{\mathrm{e}^{-\mathrm{i} \omega N}}{\omega^{2}} \int_{-\infty}^{\infty} \psi((\lambda+\omega) N) \psi((\omega-\lambda) N) \lambda^{2} g_{0}(\lambda) \mathrm{d}(N \lambda) \\
& \quad=\mathrm{O}\left(\psi^{2}(\omega N) g_{0}(\omega)\right)=\mathrm{O}(1 / N) \\
& \frac{N}{\omega^{2}} \int_{-\infty}^{\infty}|B(\lambda N)|^{2} \lambda^{2} g_{0}(\lambda) \mathrm{d} \lambda \rightarrow \frac{1}{\omega^{2}} \lim _{\lambda \rightarrow 0}\left(\lambda^{2} g_{0}(\lambda)\right)=0
\end{aligned}
$$

and

$$
\begin{aligned}
& \frac{N}{\omega^{2}} \int_{-\infty}^{\infty} B((\lambda+\omega) N) B(\lambda N) \lambda^{2} g_{0}(\lambda) \mathrm{d} \lambda \\
& \quad=\mathrm{O}\left(\frac{\psi(\omega N)}{\omega^{2}} \lim _{\lambda \rightarrow 0}\left(\lambda^{2} g_{0}(\lambda)\right)\right)=\mathrm{o}(1 / N)
\end{aligned}
$$

for all $\omega \neq 0$.

Analogous to (A.6)-(A.8), we can show that as $N \rightarrow \infty$

$$
\begin{aligned}
& \frac{1}{N^{2}} \int_{0}^{N} \int_{0}^{N} \int_{0}^{N} \int_{0}^{N}\left\{r_{X}(t, u) r_{X}(s, v)\right\} \mathrm{e}^{\mathrm{i} \omega(t-s)+\mathrm{i} \omega(u-v)} \mathrm{d} s \mathrm{~d} t \mathrm{~d} u \mathrm{~d} v \\
& =\mathrm{O}\left(1 / N^{2}\right)+\mathrm{o}(1 / N)+\mathrm{o}(1)
\end{aligned}
$$

On the other hand, similar to (A.1)-(A.3) we have as $N \rightarrow \infty$

$$
\begin{aligned}
\frac{1}{N^{2}} & \int_{0}^{N} \int_{0}^{N} \int_{0}^{N} \int_{0}^{N}\left\{r_{X}(t, v) r_{X}(s, u)\right\} \mathrm{e}^{\mathrm{i} \omega(t-v)+\mathrm{i} \omega(u-s)} \mathrm{d} s \mathrm{~d} t \mathrm{~d} u \mathrm{~d} v \\
= & \left(\frac{N}{2 \pi} \int_{-\infty}^{\infty}|B(\omega N)-B((\omega-\lambda) N)|^{2} g_{0}(\lambda) \mathrm{d} \lambda\right) \\
& \times\left(\frac{N}{2 \pi} \int_{-\infty}^{\infty}|B(\omega N)-B((\omega+\lambda) N)|^{2} g_{0}(\lambda) \mathrm{d} \lambda\right)=g^{2}\left(\omega, \mu_{0}\right)+\mathrm{o}(1)
\end{aligned}
$$

for all $\omega \neq 0$.

Thus, the proof of (2.5) follows from (A.5). Similar to (A.5)-(A.8), we can show that as $N \rightarrow \infty$

$$
\begin{aligned}
& \frac{1}{N} \int_{0}^{N} \int_{0}^{N} r_{X}(t, u) \mathrm{e}^{\mathrm{i} x t+\mathrm{i} y u} \mathrm{~d} t \mathrm{~d} u \\
& =\frac{-N}{2 \pi x y} \int_{-\infty}^{\infty} \lambda^{2}\left(B((\lambda+y) N)+\mathrm{e}^{\mathrm{i} y N} B(\lambda N)\right)(B((x-\lambda) N) \\
& \left.\quad+\mathrm{e}^{\mathrm{i} x N} B(-\lambda N)\right) g_{0}(\lambda) \mathrm{d} \lambda \rightarrow 0
\end{aligned}
$$


and

$$
\begin{aligned}
& \frac{1}{N} \int_{0}^{N} \int_{0}^{N} r_{X}(s, v) \mathrm{e}^{-\mathrm{i} x s-\mathrm{i} y v} \mathrm{~d} s \mathrm{~d} v \\
& =\frac{-N}{2 \pi x y} \int_{-\infty}^{\infty} \lambda^{2}\left(B((\lambda-y) N)+\mathrm{e}^{-\mathrm{i} y N} B(\lambda N)\right)(B((-x-\lambda) N) \\
& \left.\quad+\mathrm{e}^{-\mathrm{i} x N} B(-\lambda N)\right) g_{0}(\lambda) \mathrm{d} \lambda \rightarrow 0
\end{aligned}
$$

for all $x \neq 0$ and $x \neq y$.

Analogously, we have as $N \rightarrow \infty$

$$
\begin{aligned}
& \frac{1}{N} \int_{0}^{N} \int_{0}^{N} r_{X}(t, v) \mathrm{e}^{\mathrm{i} x t-\mathrm{i} y v} \mathrm{~d} t \mathrm{~d} v \\
& =\frac{N}{2 \pi x y} \int_{-\infty}^{\infty} \lambda^{2}\left(B((\lambda-y) N)+\mathrm{e}^{-\mathrm{i} y N} B(\lambda N)\right)(B((x-\lambda) N) \\
& \left.\quad+\mathrm{e}^{\mathrm{i} x N} B(-\lambda N)\right) g_{0}(\lambda) \mathrm{d} \lambda \rightarrow 0
\end{aligned}
$$

and

$$
\begin{aligned}
& \frac{1}{N} \int_{0}^{N} \int_{0}^{N} r_{X}(s, u) \mathrm{e}^{-\mathrm{i} x s+\mathrm{i} y u} \mathrm{~d} s \mathrm{~d} u \\
& =\frac{N}{2 \pi x y} \int_{-\infty}^{\infty} \lambda^{2}\left(B((y-\lambda) N)+\mathrm{e}^{\mathrm{i} y N} B(-\lambda N)\right)(B((\lambda-x) N) \\
& \left.\quad+\mathrm{e}^{-\mathrm{i} x N} B(\lambda N)\right) g_{0}(\lambda) \mathrm{d} \lambda \rightarrow 0
\end{aligned}
$$

for all $x \neq 0$ and $x \neq y$.

Thus, (A.9) and (A.10) imply that as $N \rightarrow \infty$

$$
\begin{aligned}
& E\left\{\left(I_{N}^{X}(x)-E\left[I_{N}^{X}(x)\right]\right)\left(I_{N}^{X}(y)-E\left[I_{N}^{X}(y)\right]\right)\right\} \\
& =\frac{1}{N^{2}} \int_{0}^{N} \int_{0}^{N} \int_{0}^{N} \int_{0}^{N} r_{X}(t, u) r_{X}(s, v) \mathrm{e}^{\mathrm{i} x t+\mathrm{i} y u} \mathrm{e}^{-\mathrm{i} x s-\mathrm{i} y v} \mathrm{~d} s \mathrm{~d} t \mathrm{~d} u \mathrm{~d} v \\
& \quad+\frac{1}{N^{2}} \int_{0}^{N} \int_{0}^{N} \int_{0}^{N} \int_{0}^{N} r_{X}(t, v) r_{X}(s, u) \mathrm{e}^{\mathrm{i} x t+\mathrm{i} y u} \mathrm{e}^{-\mathrm{i} x s-\mathrm{i} y v} \mathrm{~d} s \mathrm{~d} t \mathrm{~d} u \mathrm{~d} v \rightarrow 0
\end{aligned}
$$

for all $x \neq 0$ and $x \neq y$. Thus the proof of (2.6) is completed.

Proof of Theorem 2.2. Before proving $\hat{\mu}_{N} \rightarrow \mu_{0}$ in probability, we show that as $N \rightarrow \infty$

$$
L_{N}^{X}(\mu) \rightarrow L^{X}(\mu)=\frac{1}{4 \pi} \int_{-\infty}^{\infty}\left\{\log (g(\omega, \mu))+\frac{g\left(\omega, \mu_{0}\right)}{g(\omega, \mu)}\right\} \frac{1}{1+\omega^{2}} \mathrm{~d} \omega
$$

for $\varepsilon \leqslant\left\|\mu-\mu_{0}\right\|<\frac{1}{4}$ with any given $\varepsilon>0$. 
In order to prove (A.11), it suffices to show that for

$$
p(\omega)=p(\omega, \mu)=\frac{1}{1+\omega^{2}} g(\omega, \mu)^{-1}=\eta^{-1} \omega^{2 \gamma}\left(1+\omega^{2}\right)^{\alpha-1} \pi(\omega)^{-1}
$$

the following

$$
\int_{-\infty}^{\infty} I_{N}^{X}(\omega) p(\omega, \mu) \mathrm{d} \omega \rightarrow \int_{-\infty}^{\infty} g\left(\omega, \mu_{0}\right) p(\omega, \mu) \mathrm{d} \omega
$$

holds in probability as $N \rightarrow \infty$. It is easy to see that (A.12) follows from

$$
\int_{-\infty}^{\infty}\left(E\left[I_{N}^{X}(\omega)\right]-g\left(\omega, \mu_{0}\right)\right) p(\omega, \mu) \mathrm{d} \omega \rightarrow 0
$$

and

$$
\int_{-\infty}^{\infty}\left(I_{N}^{X}(\omega)-E\left[I_{N}^{X}(\omega)\right]\right) p(\omega, \mu) \mathrm{d} \omega \rightarrow 0
$$

in probability for $\varepsilon \leqslant\left\|\mu-\mu_{0}\right\|<\frac{1}{4}$ with any given $\varepsilon>0$.

The proof of (A.13) follows from (A.3), Condition 2.1(iii) and

$$
\int_{-\infty}^{\infty} g_{0}(\omega) p(\omega) \mathrm{d} \omega=\int_{-\infty}^{\infty} \frac{g\left(\omega, \mu_{0}\right)}{g(\omega, \mu)} \frac{1}{1+\omega^{2}} \mathrm{~d} \omega<\infty
$$

for $\varepsilon \leqslant\left\|\mu-\mu_{0}\right\|<\frac{1}{4}$, where $g_{0}(\omega)=g\left(\omega, \mu_{0}\right)$.

In order to prove (A.14), it suffices to show that as $N \rightarrow \infty$

$$
\begin{aligned}
& 4 \pi^{2} N^{2} E\left\{\int_{-\infty}^{\infty}\left(I_{N}^{X}(\omega)-E\left[I_{N}^{X}(\omega)\right]\right) p(\omega) \mathrm{d} \omega\right\}^{2} \\
& =4 \pi^{2} N^{2} \int_{-\infty}^{\infty} \int_{-\infty}^{\infty} E\left\{\left(I_{N}^{X}(x)-E\left[I_{N}^{X}(x)\right]\right)\left(I_{N}^{X}(y)-E\left[I_{N}^{X}(y)\right]\right)\right\} p(x) p(y) \mathrm{d} x \mathrm{~d} y \\
& =\int_{-\infty}^{\infty} \int_{-\infty}^{\infty}\left(\int_{0}^{N} \int_{0}^{N} \int_{0}^{N} \int_{0}^{N} r_{X}(t, u) r_{X}(s, v) \mathrm{e}^{\mathrm{i} x t+\mathrm{i} y u} \mathrm{e}^{-\mathrm{i} x s-\mathrm{i} y v} \mathrm{~d} s \mathrm{~d} t \mathrm{~d} u \mathrm{~d} v\right) p(x) p(y) \mathrm{d} x \mathrm{~d} y \\
& +\int_{-\infty}^{\infty} \int_{-\infty}^{\infty}\left(\int_{0}^{N} \int_{0}^{N} \int_{0}^{N} \int_{0}^{N} r_{X}(t, v) r_{X}(s, u) \mathrm{e}^{\mathrm{i} x t+\mathrm{i} y u} \mathrm{e}^{-\mathrm{i} x s-\mathrm{i} y v} \mathrm{~d} s \mathrm{~d} t \mathrm{~d} u \mathrm{~d} v\right) p(x) p(y) \mathrm{d} x \mathrm{~d} y \\
& =\mathrm{o}\left(N^{2}\right) .
\end{aligned}
$$

Similar to (A.6)-(A.10), and using (A.15) we can show that (A.16) holds.

We now complete the proof of Theorem 2.2. It follows from (A.12) that in probability

$$
L_{N}^{X}(\mu)-L_{N}^{X}\left(\mu_{0}\right) \rightarrow K\left(\mu, \mu_{0}\right)
$$

where for $\mu \neq \mu_{0}$

$$
K\left(\mu, \mu_{0}\right)=\frac{1}{4 \pi} \int_{-\infty}^{\infty}\left\{\frac{g\left(\omega, \mu_{0}\right)}{g(\omega, \mu)}-1-\log \left(\frac{g\left(\omega, \mu_{0}\right)}{g(\omega, \mu)}\right)\right\} \frac{\mathrm{d} \omega}{1+\omega^{2}}>0
$$


using the fact that for $x>0$ and $x \neq 1$

$$
x-1>\log (x) \text {. }
$$

Thus, for any given $\varepsilon>0$

$$
\lim \inf _{N \rightarrow \infty} \inf _{\left\|\mu-\mu_{0}\right\| \geqslant \varepsilon}\left(L_{N}^{X}(\mu)-L_{N}^{X}\left(\mu_{0}\right)\right)>0
$$

in probability. This implies that $\hat{\mu}_{N} \rightarrow \mu_{0}$ holds in probability. Thus the proof of Theorem 2.2 is completed.

Proof of Theorem 2.3. It follows from

$$
I_{N}^{Y}(\omega)=\frac{1}{2 \pi N} \int_{0}^{N} \int_{0}^{N} Y(s) Y(t) \mathrm{e}^{\mathrm{i}(s-t) \omega} \mathrm{d} s \mathrm{~d} t
$$

that

$$
\begin{aligned}
E\left[I_{N}^{Y}(\omega)\right]-\phi\left(\omega, \theta_{0}\right) & =\frac{1}{2 \pi} \int_{-\infty}^{\infty}\left[\phi_{0}(\lambda)-\phi_{0}(\omega)\right] \psi^{2}((\lambda-\omega) N) \mathrm{d}(N \lambda) \\
& =\frac{1}{2 \pi} \int_{-\infty}^{\infty}\left[\phi_{0}(\omega+u / N)-\phi_{0}(\omega)\right] \psi^{2}(u) \mathrm{d} u \\
& =\frac{1}{2 \pi}\left\{\int_{-\infty}^{-N}+\int_{-N}^{N}+\int_{N}^{\infty}\right\}\left[\phi_{0}(\omega+u / N)-\phi_{0}(\omega)\right] \psi^{2}(u) \mathrm{d} u \\
& \equiv C_{1 N}+C_{2 N}+C_{3 N},
\end{aligned}
$$

where $\phi_{0}(\omega)=\phi\left(\omega, \theta_{0}\right)$.

It is obvious that for all $\omega \neq 0$

$$
\begin{aligned}
C_{1 N} & =\int_{-\infty}^{-N}\left[\phi_{0}(\omega+u / N)-\phi_{0}(\omega)\right] \psi^{2}(u) \mathrm{d} u=\mathrm{O}\left(\phi_{0}(\omega) \int_{-\infty}^{-N} u^{-2} \mathrm{~d} u\right) \\
& =\mathrm{O}\left(\frac{\phi_{0}(\omega)}{N}\right)
\end{aligned}
$$

and

$$
\begin{aligned}
C_{3 N} & =\int_{N}^{\infty}\left[\phi_{0}(\omega+u / N)-\phi_{0}(\omega)\right] \psi^{2}(u) \mathrm{d} u=\mathrm{O}\left(\phi_{0}(\omega) \int_{N}^{\infty} u^{-2} \mathrm{~d} u\right) \\
& =\mathrm{O}\left(\frac{\phi_{0}(\omega)}{N}\right)
\end{aligned}
$$

as $N \rightarrow \infty$.

For $C_{2 N}$, a second-order Taylor expansion implies that as $N \rightarrow \infty$

$$
\begin{aligned}
C_{2 N} & =\int_{-N}^{N}\left[\phi_{0}(\omega+u / N)-\phi_{0}(\omega)\right] \psi^{2}(u) \mathrm{d} u \\
& =\int_{-N}^{N}\left\{\phi_{0}^{\prime}(\omega)(u / N)+\phi_{0}^{\prime \prime}(\xi)\left(u^{2} / 2 N^{2}\right)\right\} \psi^{2}(u) \mathrm{d} u=\mathrm{O}\left(\frac{\phi_{0}^{\prime \prime}(\omega)}{N^{2}}\right)
\end{aligned}
$$


for all $\omega \neq 0$, where $\xi$ lies between $\omega$ and $\omega+u / N$ and $\phi^{\prime \prime}(\omega)$ is the second derivative of $\phi_{0}(\omega)$ with respect to $\omega$.

This completes the proof of (2.9).

In order to prove $(2.10)$, it suffices to show that

$$
E\left\{I_{N}^{Y}(\omega)-E\left[I_{N}^{Y}(\omega)\right]\right\}^{2}=\phi^{2}\left(\omega, \theta_{0}\right)+\mathrm{O}\left(1 / N^{2}\right) .
$$

Obviously,

$$
\begin{aligned}
4 & \pi^{2} N^{2} E\left\{I_{N}^{Y}(\omega)-E\left[I_{N}^{Y}(\omega)\right]\right\}^{2} \\
& =\int_{0}^{N} \int_{0}^{N} \int_{0}^{N} \int_{0}^{N}\left\{r_{Y}(s, t, u, v)-r_{Y}(s, t) r_{Y}(u, v)\right\} \mathrm{e}^{\mathrm{i} \omega(t-s)+\mathrm{i} \omega(u-v)} \mathrm{d} s \mathrm{~d} t \mathrm{~d} u \mathrm{~d} v .
\end{aligned}
$$

Since $\{Y(s),-\infty<s<\infty\}$ is Gaussian with $Y(0)=0$, we have

$$
r_{Y}(s, t, u, v)-r_{Y}(s, t) r_{Y}(u, v)=r_{Y}(t, u) r_{Y}(s, v)+r_{Y}(t, v) r_{Y}(s, u) .
$$

Thus, it follows from (A.17) that

$$
\begin{aligned}
4 \pi^{2} E & \left\{I_{N}^{Y}(\omega)-E\left[I_{N}^{Y}(\omega)\right]\right\}^{2} \\
= & \frac{1}{N^{2}} \int_{0}^{N} \int_{0}^{N} \int_{0}^{N} \int_{0}^{N} r_{y}(t, u) r_{Y}(s, v) \mathrm{e}^{\mathrm{i}(t+u) \omega} \mathrm{e}^{-\mathrm{i}(s+v) \omega} \mathrm{d} s \mathrm{~d} t \mathrm{~d} u \mathrm{~d} v \\
& +\frac{1}{N^{2}} \int_{0}^{N} \int_{0}^{N} \int_{0}^{N} \int_{0}^{N} r_{Y}(t, v) r_{Y}(s, u) \mathrm{e}^{\mathrm{i}(t-v) \omega} \mathrm{e}^{\mathrm{i}(u-s) \omega} \mathrm{d} s \mathrm{~d} t \mathrm{~d} u \mathrm{~d} v .
\end{aligned}
$$

We now look at the first component of (A.18). For all $\omega \neq 0$, we have

$$
\begin{aligned}
& \frac{1}{N} \int_{0}^{N} \int_{0}^{N} r_{Y}(t, u) \mathrm{e}^{\mathrm{i}(t+u) \omega} \mathrm{d} t \mathrm{~d} u \\
& =\frac{1}{N} \int_{-\infty}^{\infty} \int_{0}^{N} \int_{0}^{N} \mathrm{e}^{\mathrm{i}(t+u) \omega} \mathrm{e}^{\mathrm{i}(u-t) \lambda} \phi_{0}(\lambda) \mathrm{d} t \mathrm{~d} u \mathrm{~d} \lambda \\
& =\mathrm{e}^{-\mathrm{i} \omega N} \int_{-\infty}^{\infty} \psi(N(2 \omega-z)) \psi(N z) \phi_{0}(\omega-z) \mathrm{d}(N z) \\
& =\mathrm{O}\left(\psi(2 \omega N) \phi_{0}(\omega)\right)=\mathrm{O}(1 / N)
\end{aligned}
$$

and

$$
\begin{aligned}
\frac{1}{N} \int_{0}^{N} \int_{0}^{N} r_{Y}(s, v) \mathrm{e}^{-\mathrm{i}(s+v) \omega} \mathrm{d} s \mathrm{~d} v & =\mathrm{e}^{\mathrm{i} \omega N} \int_{-\infty}^{\infty} \psi(N(2 \omega+x)) \psi(N x) \phi_{0}(\omega+x) \mathrm{d}(N x) \\
& =\mathrm{O}\left(\psi(2 \omega N) \phi_{0}(\omega)\right)=\mathrm{O}(1 / N)
\end{aligned}
$$

as $N \rightarrow \infty$. 
Analogous to (A.19) and (A.20), we can estimate that the second component of (A.18) is

$$
\begin{aligned}
& \frac{1}{N^{2}} \int_{0}^{N} \int_{0}^{N} \int_{0}^{N} \int_{0}^{N} r_{Y}(t, v) r_{Y}(s, u) \mathrm{e}^{\mathrm{i}(t-v) \omega} \mathrm{e}^{\mathrm{i}(u-s) \omega} \mathrm{d} s \mathrm{~d} t \mathrm{~d} u \mathrm{~d} v \\
& \quad=\left(\int_{-\infty}^{\infty} \psi^{2}((\omega-\lambda) N) \phi_{0}(\lambda) \mathrm{d} \lambda\right)\left(\int_{-\infty}^{\infty} \psi^{2}((\omega+\lambda) N) \phi_{0}(\lambda) \mathrm{d} \lambda\right) \rightarrow \phi^{2}\left(\omega, \theta_{0}\right)
\end{aligned}
$$

as $N \rightarrow \infty$.

This completes the proof of (2.10). Analogously, we can prove (2.11) and therefore we complete the proof of Theorem 2.3.

Proof of Theorem 2.4. Before proving $\hat{\theta}_{N} \rightarrow \theta_{0}$ with probability one, we show that as $N \rightarrow \infty$

$$
L_{N}^{Y}(\theta) \rightarrow L^{Y}(\theta)=\frac{1}{4 \pi} \int_{-\infty}^{\infty}\left\{\log (\phi(\omega, \theta))+\frac{\phi\left(\omega, \theta_{0}\right)}{\phi(\omega, \theta)}\right\} \frac{\mathrm{d} \omega}{1+\omega^{2}}
$$

holds with probability one for $\delta \leqslant\left\|\theta-\theta_{0}\right\|<\frac{1}{4}$ with any given $\delta>0$.

In order to prove (A.21), it suffices to show that for

$$
q(\omega)=q(\omega, \theta)=\frac{1}{1+\omega^{2}} \phi(\omega)^{-1}=(16 \eta)^{-1} \omega^{2 \beta}\left(1+\omega^{2}\right)^{\alpha-1}\left(\frac{\sin (\omega / 4)}{\omega / 4}\right)^{-4} \pi^{-1}(\omega)
$$

the following

$$
\int_{-\infty}^{\infty} I_{N}^{Y}(\omega) q(\omega, \theta) \mathrm{d} \omega \rightarrow \int_{-\infty}^{\infty} \phi\left(\omega, \theta_{0}\right) q(\omega, \theta) \mathrm{d} \omega
$$

holds with probability one as $N \rightarrow \infty$. It is easy to see that (A.22) follows from

$$
\int_{-\infty}^{\infty}\left(E\left[I_{N}^{Y}(\omega)\right]-\phi\left(\omega, \theta_{0}\right)\right) q(\omega, \theta) \mathrm{d} \omega \rightarrow 0
$$

and

$$
\int_{-\infty}^{\infty}\left(I_{N}^{Y}(\omega)-E\left[I_{N}^{Y}(\omega)\right]\right) q(\omega, \theta) \mathrm{d} \omega \rightarrow 0
$$

with probability one for $\delta \leqslant\left\|\theta-\theta_{0}\right\|<\frac{1}{4}$ with any given $\delta>0$. We first prove (A.23).

It follows from the definition of $I_{N}^{Y}(\omega)$ that as $N \rightarrow \infty$

$$
\int_{-\infty}^{\infty} E\left[I_{N}^{Y}(\omega)\right] q(\omega, \theta) \mathrm{d} \omega \rightarrow \int_{-\infty}^{\infty} \phi\left(\omega, \theta_{0}\right) q(\omega, \theta) \mathrm{d} \omega
$$

using for $\delta \leqslant\left\|\theta-\theta_{0}\right\|<\frac{1}{4}$,

$$
\int_{-\infty}^{\infty} \phi_{0}(\omega) q(\omega) \mathrm{d} \omega<\infty
$$

due to Condition 2.1(iii).

Hence, (A.25) implies (A.23). In order to prove (A.24), it suffices to show that as $N \rightarrow \infty$

$$
E\left\{\int_{-\infty}^{\infty}\left(I_{N}^{Y}(\omega)-E\left[I_{N}^{Y}(\omega)\right]\right) q(\omega) \mathrm{d} \omega\right\}^{2}=\mathrm{O}\left(\frac{1}{N^{2}}\right)
$$


The proof of (A.26) is similar to that of (A.6) of Gao et al. (2001).

The remainder of the proof of Theorem 2.4(i) is similar to that of Theorem 2.2. We shall not repeat the details here.

We now prove Theorem 2.4(ii). Note that

$$
\left.\frac{\partial L_{N}^{Y}(\theta)}{\partial \theta}\right|_{\theta=\hat{\theta}_{N}}-\left.\frac{\partial L_{N}^{Y}(\theta)}{\partial \theta}\right|_{\theta=\theta_{0}}=\left[\left.\frac{\partial^{2}}{\partial \theta^{2}} L_{N}^{Y}(\theta)\right|_{\theta=\theta_{N}^{*}}\right]\left(\hat{\theta}_{N}-\theta_{0}\right),
$$

where $\left|\theta_{N}^{*}-\theta_{0}\right| \leqslant\left|\hat{\theta}_{N}-\theta_{0}\right|$. Since $\theta_{0}$ is in the interior of $\Theta$, Theorem 2.4(i) implies that $\hat{\theta}_{N}$ is in the interior of $\Theta$ for large $N$. Since $\hat{\theta}_{N}$ minimizes $L_{N}^{Y}(\theta)$, it follows that $\partial L_{N}^{Y}(\theta) /\left.\partial \theta\right|_{\theta=\hat{\theta}_{N}}=0$ for large $N$. Thus for large $N$

$$
\left.\frac{\partial L_{N}^{Y}(\theta)}{\partial \theta}\right|_{\theta=\theta_{0}}=\left[-\left.\frac{\partial^{2}}{\partial \theta^{2}} L_{N}^{Y}(\theta)\right|_{\theta=\theta_{N}^{*}}\right]\left(\hat{\theta}_{N}-\theta_{0}\right) .
$$

Thus, in order to prove that as $N \rightarrow \infty$

$$
\sqrt{N}\left(\hat{\theta}_{N}-\theta_{0}\right) \rightarrow_{\mathrm{D}} \mathrm{N}\left(0, \Gamma^{-1}\left(\theta_{0}\right)\right)
$$

it suffices to show that as $N \rightarrow \infty$

$$
\sqrt{N} \int_{-\infty}^{\infty}\left\{I_{N}^{Y}(\omega)-E\left[I_{N}^{Y}(\omega)\right]\right\} D(\omega, \theta) \mathrm{d} \omega \rightarrow \mathrm{N}\left(0,16 \pi^{2} \Gamma\left(\theta_{0}\right)\right),
$$

where

$$
\Gamma(\theta)=\frac{1}{4 \pi} \int_{-\infty}^{\infty} \frac{1}{\left(1+\omega^{2}\right)^{2}}\left\{\frac{\partial}{\partial \theta} \log (\phi(\omega, \theta))\right\}\left\{\frac{\partial}{\partial \theta} \log (\phi(\omega, \theta))\right\}^{T} \mathrm{~d} \omega
$$

and

$$
\begin{aligned}
D(\omega, \theta) & =-\frac{1}{1+\omega^{2}} \frac{\partial \phi^{-1}(\omega, \theta)}{\partial \theta} \\
& =-\frac{1}{1+\omega^{2}}\left(\frac{\partial \phi^{-1}(\omega, \theta)}{\partial \alpha}, \frac{\partial \phi^{-1}(\omega, \theta)}{\partial \beta}, \frac{\partial \phi^{-1}(\omega, \theta)}{\partial \eta}\right)^{T} \\
& \equiv\left(D_{1}(\omega, \theta), D_{2}(\omega, \theta), D_{3}(\omega, \theta)\right)^{T}
\end{aligned}
$$

To prove (A.27), it suffices to show that for any fixed $c=\left(c_{1}, c_{2}, c_{3}\right)^{\tau}$,

$$
\sqrt{N} \int_{-\infty}^{\infty}\left\{I_{N}^{Y}(\omega)-E\left[I_{N}^{Y}(\omega)\right]\right\} H(\omega, \theta) \mathrm{d} \omega \rightarrow N\left(0, \sigma^{2}\left(\theta_{0}\right)\right)
$$

as $N \rightarrow \infty$, where $H(\omega, \theta)=\sum_{j=1}^{3} c_{j} D_{j}(\omega, \theta)$ and

$$
\sigma^{2}(\theta)=4 \pi \int_{-\infty}^{\infty} \frac{1}{\left(1+\omega^{2}\right)^{2}}\left(\sum_{j=1}^{3} c_{j} \frac{\partial}{\partial \theta_{j}} \log (\phi(\omega, \theta))\right)^{2} \mathrm{~d} \omega,
$$

in which $\theta_{1}=\alpha, \theta_{2}=\beta$ and $\theta_{3}=\gamma$. 
Without loss of generality, we assume that there is a measurable function $k(\omega, \theta)$ such that $k(\omega, \theta)$ is symmetric in $\omega$ and

$$
H(\omega, \theta)=\sum_{j=1}^{3} c_{j} D_{j}(\omega, \theta)=k^{2}(\omega, \theta) \geqslant 0 .
$$

Otherwise, one needs only to consider the positive and negative parts of $H(\omega, \theta)$ separately.

Let $k(\omega)=k(\omega, \theta)$,

$$
G_{N}(\omega)=\frac{k(\omega)}{\sqrt{2 \pi}} \int_{0}^{N} \mathrm{e}^{\mathrm{i} \omega t} Y(t) \mathrm{d} t \text { and } R_{N}(u, v)=E\left[G_{N}(u) G_{N}(-v)\right] .
$$

For any function $l \in L^{2}(-\infty, \infty)$, define the integral operator $\mathscr{L}_{N} \in L^{2}(-\infty, \infty) \rightarrow$ $L^{2}(-\infty, \infty)$ by

$$
\mathscr{L}_{N} l=\int_{-\infty}^{\infty} R_{N}(u, v) l(v) \mathrm{d} v .
$$

Let $\lambda_{k}^{(N)}$ be the eigenvalues of $\mathscr{L}_{N}$. Then it follows from the Karhunen-Loéve expansion (e.g. Yeh, 1973, Theorem 19.4) that

$$
\int_{-\infty}^{\infty}\left|G_{N}(\omega)\right|^{2} \mathrm{~d} \omega=\sum_{i=1}^{\infty} \lambda_{i}^{(N)}\left(G_{i}^{(N)}\right)^{2}
$$

where for any $N,\left\{G_{i}^{(N)}: i \geqslant 1\right\}$ is a sequence of independent $\mathrm{N}(0,1)$ random variables.

It follows from (A.29) that

$$
m_{N}=\int_{-\infty}^{\infty} E\left[\left|G_{N}(u)\right|^{2}\right] \mathrm{d} u=\int_{-\infty}^{\infty} R_{N}(u, u) \mathrm{d} u=\sum_{i=1}^{\infty} \lambda_{i}^{(N)}
$$

and

$$
\begin{aligned}
\Delta_{N}^{2} & =\operatorname{Var}\left(\int_{-\infty}^{\infty}\left|G_{N}(\omega)\right|^{2} \mathrm{~d} \omega\right) \\
& =2 \int_{-\infty}^{\infty} \int_{-\infty}^{\infty} R_{N}^{2}(u, v) \mathrm{d} u \mathrm{~d} v=2 \sum_{i=1}^{\infty}\left(\lambda_{i}^{(N)}\right)^{2} .
\end{aligned}
$$

Note that

$$
\begin{aligned}
& \sqrt{N} \int_{-\infty}^{\infty}\left\{I_{N}^{Y}(\omega)-E\left[I_{N}^{Y}(\omega)\right]\right\} H(\omega, \theta) \mathrm{d} \omega \\
& =\frac{1}{\sqrt{N}} \int_{-\infty}^{\infty}\left(\left|G_{N}(\omega)\right|^{2}-E\left[\left|G_{N}(\omega)\right|^{2}\right]\right) \mathrm{d} \omega
\end{aligned}
$$

In order to prove (A.28), in view of (A.29)-(A.31), it suffices to show that as $N \rightarrow \infty$

$$
\frac{\max _{k} \lambda_{k}^{(N)}}{\sqrt{\sum_{k=1}^{\infty}\left(\lambda_{k}^{(N)}\right)^{2}}} \rightarrow 0
$$


To prove (A.32), it suffices to show that as $N \rightarrow \infty$

$$
\int_{-\infty}^{\infty} \int_{-\infty}^{\infty} R_{N}^{2}(u, v) \mathrm{d} u \mathrm{~d} v \rightarrow \infty
$$

and

$$
\frac{\int_{-\infty}^{\infty}\left(\int_{-\infty}^{\infty} R_{N}(u, v) l(v) \mathrm{d} v\right)^{2} \mathrm{~d} u}{\int_{-\infty}^{\infty} \int_{-\infty}^{\infty} R_{N}^{2}(u, v) \mathrm{d} u \mathrm{~d} v} \rightarrow 0
$$

Similar to (A.19), it can be shown that as $N \rightarrow \infty$

$$
\begin{aligned}
R_{N}(u, v) & =E\left[G_{N}(u) G_{N}(-v)\right] \\
& =\frac{k(u) k(v)}{2 \pi} \int_{-\infty}^{\infty}\left(\int_{0}^{N} \int_{0}^{N} \mathrm{e}^{\mathrm{i} t(v-\lambda)} \mathrm{e}^{-\mathrm{i} s(u-\lambda)} \mathrm{d} s \mathrm{~d} t\right) \phi_{0}(\lambda) \mathrm{d} \lambda \\
& =\frac{k(u) k(v) N^{2}}{2 \pi} \int_{-\infty}^{\infty} B(N(v-\lambda)) B(-N(u-\lambda)) \phi_{0}(\lambda) \mathrm{d} \lambda \\
& =N(1+\mathrm{o}(1)) B(-N(u-v)) \phi_{0}(v) k(u) k(v),
\end{aligned}
$$

where $B(x)=\left(\mathrm{e}^{\mathrm{i} x}-1\right) / \mathrm{i} x$.

Thus, as $N \rightarrow \infty$

$$
\begin{aligned}
& \int_{-\infty}^{\infty} \int_{-\infty}^{\infty} R_{N}^{2}(u, v) \mathrm{d} u \mathrm{~d} v \\
& =\frac{2 \pi N^{2}(1+\mathrm{o}(1))}{2 \pi} \int_{-\infty}^{\infty}\left(\int_{-\infty}^{\infty}|B(N(u-v))|^{2} \phi_{0}^{2}(v) k^{2}(v) \mathrm{d} v\right) k^{2}(u) \mathrm{d} u \\
& =2 \pi N(1+\mathrm{o}(1)) \int_{-\infty}^{\infty} \phi_{0}^{2}(u) H^{2}(u) \mathrm{d} u \rightarrow \infty
\end{aligned}
$$

This implies that (A.33) holds.

Similarly, as $N \rightarrow \infty$

$$
\begin{aligned}
\int_{-\infty}^{\infty} R_{N}(u, v) l(v) \mathrm{d} u \mathrm{~d} v & =\frac{2 \pi N^{2}(1+\mathrm{o}(1))}{2 \pi} \int_{-\infty}^{\infty} B(-N(u-v)) \phi_{0}(v) h(u) h(v) l(v) \mathrm{d} v \\
& =2 \pi \phi_{0}(u) l(u) H(u)(1+\mathrm{o}(1)) .
\end{aligned}
$$

Therefore, as $N \rightarrow \infty$

$$
\begin{aligned}
& \int_{-\infty}^{\infty}\left(\int_{-\infty}^{\infty} R_{N}(u, v) l(v) \mathrm{d} u \mathrm{~d} v\right)^{2} \mathrm{~d} u \\
& =4 \pi^{2}(1+\mathrm{o}(1)) \int_{-\infty}^{\infty} \phi_{0}^{2}(u) l^{2}(u) H^{2}(u) \mathrm{d} u<\infty
\end{aligned}
$$

Eqs. (A.35) and (A.36) imply that (A.34) holds. Therefore, the asymptotic normality of Theorem 2.4(ii) is proved. 
Proof of Theorem 2.5. For any fixed $\omega$, let

$$
A(\omega)=\frac{1}{\sqrt{2 \pi N}} \int_{0}^{N} \cos (\omega t) Y(t) \mathrm{d} t, \quad B(\omega)=\frac{1}{\sqrt{2 \pi N}} \int_{0}^{N} \sin (\omega t) Y(t) \mathrm{d} t
$$

and

$$
C(\omega)=\frac{1}{\sqrt{2 \pi N}} \int_{0}^{N} \mathrm{e}^{-\mathrm{i} \omega t} Y(t) \mathrm{d} t .
$$

Similar to (A.19) and (A.20), we have for any fixed $\omega$ and $\lambda$

$$
\begin{aligned}
E[C(\omega) C(\lambda)] & =\frac{1}{2 \pi N} \int_{0}^{N} \int_{0}^{N} \mathrm{e}^{-\mathrm{i} \omega s} \mathrm{e}^{-\mathrm{i} \lambda t} E[Y(s) Y(t)] \mathrm{d} s \mathrm{~d} t \\
& =\frac{1}{2 \pi N} \int_{0}^{N} \int_{0}^{N} \mathrm{e}^{-\mathrm{i} \omega s} \mathrm{e}^{-\mathrm{i} \lambda t}\left(\int_{-\infty}^{\infty} \mathrm{e}^{\mathrm{i} x(t-s)} \phi(x) \mathrm{d} x\right) \mathrm{d} s \mathrm{~d} t \\
& =\frac{\mathrm{e}^{-(\lambda+\omega) N \mathrm{i} / 2}}{2 \pi} \int_{-\infty}^{\infty} \psi((\omega+x) N) \psi((\lambda-x) N) \phi(x) \mathrm{d}(N x) .
\end{aligned}
$$

Note that

$$
\begin{aligned}
& A(\omega) A(\lambda)=\frac{1}{2} \operatorname{Re}(C(\omega) C(\lambda)+C(\omega) C(-\lambda)), \\
& B(\omega) B(\lambda)=\frac{1}{2} \operatorname{Re}(C(\omega) C(-\lambda)-C(\omega) C(\lambda))
\end{aligned}
$$

and

$$
A(\omega) B(\lambda)=\frac{1}{2} \operatorname{Im}(C(\omega) C(-\lambda)-C(\omega) C(\lambda)) .
$$

We now have

$$
\begin{aligned}
E[A(\omega) A(\lambda)]= & \frac{1}{4 \pi} \cos ((\omega+\lambda) N / 2) \int_{-\infty}^{\infty} \psi((\omega+x) N) \psi((\lambda-x) N) \phi(x) \mathrm{d}(N x) \\
& +\frac{1}{4 \pi} \cos ((\omega-\lambda) N / 2) \int_{-\infty}^{\infty} \psi((\omega+x) N) \psi((\lambda+x) N) \phi(x) \mathrm{d}(N x) \\
E[B(\omega) B(\lambda)]= & \frac{1}{4 \pi} \cos ((\omega-\lambda) N / 2) \int_{-\infty}^{\infty} \psi((\omega+x) N) \psi((\lambda+x) N) \phi(x) \mathrm{d}(N x) \\
& -\frac{1}{4 \pi} \cos ((\omega+\lambda) N / 2) \int_{-\infty}^{\infty} \psi((\omega+x) N) \psi((\lambda-x) N) \phi(x) \mathrm{d}(N x)
\end{aligned}
$$

and

$$
\begin{aligned}
E[A(\omega) B(\lambda)]= & \frac{1}{4 \pi} \sin ((\omega-\lambda) N / 2) \int_{-\infty}^{\infty} \psi((\omega+x) N) \psi((\lambda+x) N) \phi(x) \mathrm{d}(N x) \\
& -\frac{1}{4 \pi} \sin ((\omega+\lambda) N / 2) \int_{-\infty}^{\infty} \psi((\omega+x) N) \psi((\lambda-x) N) \phi(x) \mathrm{d}(N x) .
\end{aligned}
$$


Thus, by the similar reason as in (A.19) and (A.20) we obtain for any fixed $\omega$ and $\lambda$

$$
E[A(\omega) B(\lambda)] \rightarrow 0
$$

and

$$
\begin{aligned}
\operatorname{Var}(A(\omega))= & \frac{1}{4 \pi} \int_{-\infty}^{\infty} \psi^{2}((\omega+x) N) \phi(x) \mathrm{d}(N x) \\
& +\frac{1}{4 \pi} \cos (\omega N) \int_{-\infty}^{\infty} \psi((\omega+x) N) \psi((\omega-x) N) \phi(x) \mathrm{d}(N x) \\
& \rightarrow \frac{1}{2} \phi\left(\omega, \theta_{0}\right)
\end{aligned}
$$

as $N \rightarrow \infty$.

Analogously,

$$
\begin{aligned}
\operatorname{Var}(B(\omega))= & \frac{1}{4 \pi} \int_{-\infty}^{\infty} \psi^{2}((\omega+x) N) \phi(x) \mathrm{d}(N x) \\
& -\frac{1}{4 \pi} \cos (\omega N) \int_{-\infty}^{\infty} \psi((\omega+x) N) \psi((\omega-x) N) \phi(x) \mathrm{d}(N x) \\
& \rightarrow \frac{1}{2} \phi\left(\omega, \theta_{0}\right)
\end{aligned}
$$

as $N \rightarrow \infty$.

Therefore, the proof of Theorem 2.5 follows from the fact that the normalized coefficients $A(\omega) / \sqrt{\phi\left(\omega, \theta_{0}\right)}$ and $B(\omega) / \sqrt{\phi\left(\omega, \theta_{0}\right)}$ are Gaussian, and asymptotically independent.

\section{References}

Anh, V., Heyde, C. (Eds.), 1999. Special issue on long-range dependence. J. Statist. Plann. Inference 80, 1. Anh, V., Angulo, J., Ruiz-Medina, M., 1999a. Possible long-range dependence in fractional random fields. J. Statist. Plann. Inference $80,95-110$.

Anh, V., Heyde, C., Tieng, Q., 1999b. Stochastic models for fractal processes. J. Statist. Plann. Inference $80,123-135$.

Baillie, R., King, M. (Eds.), 1996. Special issue of journal of econometrics. Annals of Econometrics 73, 1.

Beran, J., 1994. Statistics for Long Memory Processes. Chapman \& Hall, London.

Brockwell, P., Davis, R., 1990. Time Series Theory and Methods. Springer, New York.

Dahlhaus, R., 1989. Efficient parameter estimation for self-similar processes. Ann. Statist. 17, 1749-1766.

Dym, H., McKean, H.P., 1976. Gaussian Process, Function Theory and the Inverse Spectral Problem. Academic Press, New York.

Fan, J., Gijbels, I., 1996. Local Polynomial Modelling and Its Applications. Chapman \& Hall, London.

Flandrin, P., 1989. On the spectrum of fractional Brownian motions. IEEE Trans. Inform. Theory 35, 197-199.

Frisch, U., 1995. Turbulence. Cambridge University Press, New York.

Gao, J., Anh, V., Heyde, C., Tieng, Q., 2001. Parameter estimation of stochastic processes with long-range dependence and intermittency. J. Time Ser. Anal. 22, 517-535.

Giraitis, L., Leipus, R., 1995. A generalized fractionally differencing approach in long-memory modelling. Liet. Mat. Rink. 35, 65-81. 